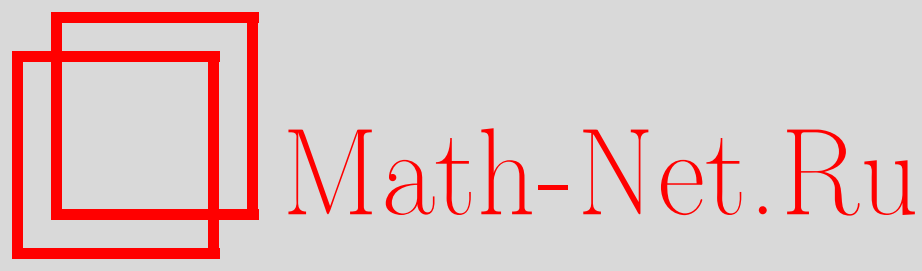

И. Г. Корепанов, Геометрические кручения и инварианты многообразий с триангулированным краем, $T M \Phi$, 2009, том 158, номер 1, 98-114

DOI: https://doi.org/10.4213/tmf6301

Использование Общероссийского математического портала Math-Net.Ru подразумевает, что вы прочитали и согласны с пользовательским соглашением http://www . mathnet.ru/rus/agreement

Параметры загрузки:

IP : 3.89 .185 .249

26 апреля 2023 г., 16:13:19

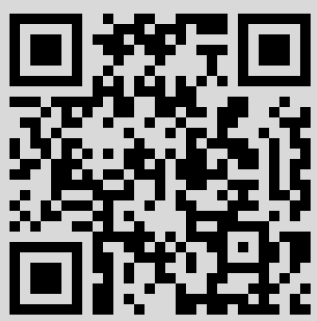




\section{ГЕОМЕТРИЧЕСКИЕ КРУЧЕНИЯ И ИНВАРИАНТЫ МНОГООБРАЗИЙ С ТРИАНГУЛИРОВАННЫМ КРАЕМ}

Геометрические кручения - это кручения ациклических комплексов векторных пространств, состоящих из дифференциалов геометрических величин, которые сопоставлены элементам триангуляции многообразия. Геометрические кручения использованы для построения инвариантов трехмерного многообразия с триангулированным краем. Эти инварианты естественно объединяются в вектор, а изменению триангуляции края соответствует линейное преобразование этого вектора. Более того, при склеивании двух многообразий по общему краю эти векторы скалярно перемножаются, т.е. подчиняются аксиомам Атьи для топологической квантовой теории поля.

Ключевые слова: топологическая квантовая теория поля, аксиомы Атьи, геометрические ациклические комплексы.

\section{1. ВВЕДЕНИЕ}

Топологическая квантовая теория поля в размерности $d$ согласно аксиомам Атьи должна сопоставлять компактному ориентированному $(d+1)$-мерному многообразию с $m$-компонентным краем "объект с $m$ ногами”, т.е. элемент тензорного произведения $m$ конечномерных векторных пространств над полем $\mathbb{C}$, каждое из которых соответствует одной из компонент края. При изменении ориентации компоненты края ее векторное пространство заменяется на двойственное (на пространство линейных функционалов над исходным пространством). Склеивание многообразий $M_{1}$ и $M_{2}$ по каким-то компонентам их краев изображается склеиванием соответствующих "ног", и упомянутые объекты перемножаются с помощью естественного спаривания векторных пространств, ибо каждая компонента края, по которой идет склеивание, имеет в $M_{1}$ и $M_{2}$ противоположные ориентации. Многообразию без края сопоставляется одно число - элемент $\mathbb{C}$. Полное изложение аксиоматики Атьи можно найти в его статье [1] или книге [2].

$\mathrm{B}$ настоящей статье предлагается способ построения теории поля с помощью "геометрических кручений", т.е. кручений ациклических комплексов векторных

*Южно-Уральский государственный университет, Челябинск, Россия. E-mail: kig@susu.ac.ru 
пространств, состоящих из дифференциалов геометрических величин, которые сопоставлены элементам триангуляции многообразия. Более конкретно, мы берем трехмерное триангулированное многообразие с краем или без края и снабжаем каждый симплекс евклидовой геометрией (в частности, ребра приобретают евклидовы длины). Эта геометрия ни в коей мере не предполагает евклидовой структуры на всем многообразии; она дает нам алгебраические величины, позволяющие построить ациклические комплексы, из которых можно получить инварианты многообразия. Впервые такой инвариант геометрического происхождения появился в работе [3], а в работах [4], [5] было показано, что стоящая за ним алгебра есть алгебра ациклических комплексов.

Ранее предлагались различные версии таких инвариантов. В частности, интерес представляют инварианты, полученные из ациклических комплексов, "скрученных" представлением фундаментальной группы многообразия. В ходе работы с ними стало ясно (хотя и не доказано в общем случае), что они связаны как с обычным, так и с неабелевым кручением Райдемайстера и скрученным полиномом Александера. Первый результат на эту тему изложен в статье [6] (без использования языка ациклических комплексов); дальнейшие результаты принадлежат Мартюшеву [7], [8] и наиболее подробно изложены в его диссертации [9].

Однако чувствовалось, что возможности геометрических кручений гораздо богаче, и к тому же оставалось желание построить инварианты, не использующие представления фундаментальной группы (которые очень часто нелегко описать). Это привело нас к изучению относителъных инвариантов. Исследование такого инварианта для линзового пространства без трубчатой окрестности неузла (т.е. наименее заузленной петли, идущей вдоль данного элемента фундаментальной группы), предпринятое в статье [10], сразу привело к нетривиальным результатам и навело на мысль сконструировать таким способом топологическую теорию поля в духе аксиом Атьи или даже непосредственно удовлетворяющую этим аксиомам. Самое замечательное, что геометрические кручения, видимо, существуют для многообразий любых размерностей (см. работы [4], [5], [11] для четырехмерного случая).

В настоящей статье мы ограничиваемся трехмерными многообразиями, которые к тому же имеют только одну компоненту края. Цель статьи - показать, как можно построить вектор инвариантов с помощью геометрических кручений и как происходит спаривание двух таких векторов при склеивании двух многообразий (в результате чего получается многообразие без края). Содержание статьи по разделам таково. В разделе 2 объясняется, как построить алгебраический комплекс и инвариант для замкнутого многообразия. Именно этот инвариант впоследствии получится при спаривании векторов инвариантов для двух многообразий с краем. Как строить эти векторы, объясняется в разделе 3. Далее в разделе 4 показывается, что при переходе к новой триангуляции края вектор инвариантов линейно преобразуется, а в разделе 5 определяется скалярное произведение двух таких векторов (для двух многообразий с одинаково триангулированными краями) и показывается, что оно равно инварианту результата склейки. Примеры, подтверждающие нетривиальность наших инвариантов, приводятся в разделе 6. В заключительном разделе 7 обсуждаются результаты статьи и перспективы дальнейших исследований. 


\section{2. АЛГЕБРАИЧЕСКИЙ КОМПЛЕКС И ПРОСТЕЙШИЙ ИНВАРИАНТ ДЛЯ ЗАМКНУТОГО ТРЕХМЕРНОГО МНОГОБРАЗИЯ}

Напомним, как строится простейший вариант алгебраического комплекса для триангулированного замкнутого ориентируемого трехмерного многообразия $M$ и соответствующий ему инвариант (по существу, это инвариант из работы [3]). Триангуляция не обязана быть строго комбинаторной, т.е. допускается как кратное вхождение симплекса в границу симплекса большей размерности, так и наличие нескольких симплексов с одинаковыми вершинами. Должно, однако, выполняться следующее условие: все четыре вершины каждого тетраэдра различны.

2.1. Построение комплекса. Каждой вершине $A$ триангуляции сопоставим три вещественных числа $x_{A}, y_{A}, z_{A}$, которые являются параметрами, входящими в наши дальнейшие построения. Будем называть $x_{A}, y_{A}, z_{A}$ невозмущенными евклидовыми координатами вершины $A$. По этим координатам определяются (невозмущенные) евклидовы объемы, приписываемые симплексам разных размерностей, например длина ребра $A B$ :

$$
l_{A B}=\sqrt{\left(x_{B}-x_{A}\right)^{2}+\left(y_{B}-y_{A}\right)^{2}+\left(z_{B}-z_{A}\right)^{2}} .
$$

Размещение вершин в евклидовом трехмерном пространстве $\mathbb{R}^{3}$ не имеет никакого отношения к топологии $M$, требуется только, чтобы координаты лежали в общем положении относительно всех последующих алгебраических конструкций. В частности, ориентированный объем каждого тетраэдра $A B C D$ равен

$$
V_{A B C D}=\frac{1}{6}|\overrightarrow{A B} \overrightarrow{A C} \overrightarrow{A D}|
$$

где определитель матрицы, составленной из трех векторов-столбцов, отличен от нуля.

Фиксируем ориентацию $M$, т.е. согласованную ориентацию всех тетраэдров; ориентация тетраэдра есть заданный порядок его вершин с точностью до четных перестановок. В дальнейшем, говоря “тетраэдр $A B C D$ ”, мы имеем в виду, что именно такой порядок его вершин задает положительную ориентацию. Тем не менее объем (2) тетраэдра $A B C D$ может принимать любой знак, и мы будем приписывать этот же знак внутренним двугранным углам тетраэдра, которые нам вскоре понадобятся.

Алгебраический комплекс состоит из векторных пространств векторов-столбцов и их линейных отображений (матриц):

$$
0 \longrightarrow \mathfrak{e}(3) \stackrel{f_{1}}{\longrightarrow}(d x) \stackrel{f_{2}}{\longrightarrow}(d l) \stackrel{f_{3}=f_{3}^{\mathrm{T}}}{\longrightarrow}(d \omega) \stackrel{f_{4}=-f_{2}^{\mathrm{T}}}{\longrightarrow}\left(d x^{*}\right) \stackrel{f_{5}=f_{1}^{\mathrm{T}}}{\longrightarrow} \mathfrak{e}(3)^{*} \longrightarrow 0 .
$$

Здесь $\mathfrak{e}(3)$ - алгебра Ли бесконечно малых движений трехмерного евклидова пространства, элемент которой мы записываем в виде вектора-столбца высоты 6 в естественном базисе из трех сдвигов и трех поворотов; $(d x)$ и $\left(d x^{*}\right)$ - векторные пространства столбцов высоты $3 N_{0}$, где $N_{0}$ - число вершин в триангуляции; $(d l)$ и $(d \omega)-$ 
векторные пространства столбцов высоты $N_{1}$, где $N_{1}$ - число ребер триангуляции. Вектор-столбец из пространства $(d x)$ записываем в виде $\left(d x_{A}, d y_{A}, d z_{A}, \ldots\right.$ $\left.\ldots, d x_{Z}, d y_{Z}, d z_{Z}\right)^{\mathrm{T}}$, где $A, \ldots, Z$ - вершины триангуляции; отображение $f_{1}$ по определению дает бесконечно малые сдвиги этих вершин из их исходных положений $\left(x_{A}, y_{A}, z_{A}\right), \ldots,\left(x_{Z}, y_{Z}, z_{Z}\right)$ под действием элемента алгебры Ли. Вектор-столбец из пространства $(d l)$ записываем в виде $\left(d l_{1}, \ldots, d l_{N_{1}}\right)^{\mathrm{T}}$, где индексы нумеруют ребра триангуляции; отображение $f_{2}$ по определению дает бесконечно малые изменения длин ребер, соответствующие данным изменениям координат вершин.

Чтобы описать отображение $f_{3}$, требуется ввести понятие угла дефекта $\omega_{i}$ вокруг данного ребра $i$. Если все длины ребер получаются из некоторых координат вершин в соответствии с равенством (1), то алгебраическая сумма двугранных углов при ребре $i$ (вспомним о знаке, который мы договорились приписывать такому углу!) $\sum_{k} \varphi_{i}^{(k)}=0(\bmod 2 \pi)$, где $k$ нумерует тетраэдры, расположенные вокруг ребра $i$. Если же длины ребер произвольно и независимо изменить, то появится угол дефекта

$$
\omega_{i} \stackrel{\text { def }}{=}-\sum_{k} \varphi_{i}^{(k)} \quad(\bmod 2 \pi) .
$$

Отображение $f_{3}$ определяет вектор бесконечно малых углов дефекта $\left(d \omega_{1}, \ldots, d \omega_{N_{1}}\right)^{\mathrm{T}}$, возникающий из данных бесконечно малых деформаций длин.

Важным свойством матрицы $f_{3}$, существенно облегчающим дальнейшее построение комплекса (3), является ее симметричность, доказанная в работе [3] ${ }^{1)}$. Поэтому можно не заботиться о приписывании геометрического смысла пространствам, обозначенным $\left(d x^{*}\right)$ и $\mathfrak{e}(3)^{*}$, а просто считать их пространствами векторов-столбцов, между которыми действуют матрицы $f_{4}$ и $f_{5}$, определенные с помощью транспонирования "зеркально симметричных" им матриц, как указано над стрелками в $(3)^{2}$.

ТЕорема 1. Последовательность (3) является алгебраическим комплексом, т.е. композиция любых двух соседних стрелок равна нулю.

Доказательство. Равенства $f_{2} \circ f_{1}=0$ и $f_{3} \circ f_{2}=0$ следуют из простых геометрических соображений: первое - из того, что движения евклидова пространства как целого не меняют длин ребер, второе - из того, что любые изменения длин ребер, обусловленные только изменениями координат вершин, оставляют углы дефекта равными нулю. Транспонируя эти равенства, получаем $f_{5} \circ f_{4}=0$ и $f_{4} \circ f_{3}=0$.

ЗАмечание 1. Комплекс (3) может и не быть ациклическим. Ациклическим является согласно результатам раздела 2 работы [4] "скрученный” комплекс, в котором задействованы универсальное накрытие триангулированного многообразия $M$, представление $\rho$ фундаментальной группы $M$ в группу движений евклидова пространства, $\pi_{1}(M) \rightarrow E(3)$, и бесконечно малые деформации представления $\rho$. Однако комплекс (3) бывает и ациклическим (если группа $\pi_{1}(M)$ конечна и, соответственно, $\rho$ не деформируется в неэквивалентное представление). Далее мы ограничимся

\footnotetext{
1) Более элегантный метод доказательства симметричности $f_{3}$ можно извлечь из раздела 4 работы [11], где речь идет об аналогичной матрице для случая четырехмерного многообразия.

2) Знак минус в определении $f_{4}$ является уточнением, которым я обязан Е. В. Мартюшеву. Этот знак будет играть важную роль в определении кручений (5) и (9) и получаемых из них инвариантов.
} 
случаем ациклического комплекса, чтобы не перегружать изложение, и кроме того, именно такой комплекс используется ниже в теореме 6 .

ЗАмЕчание 2. Ацикличность может нарушаться в членах $(d l)$ и $(d \omega)$. С другой стороны, любые изменения координат, сохраняющие все длины, получаются движением евклидова пространства, что приводит к ацикличности в члене $(d x)$, и с помощью симметрии в $\left(d x^{*}\right)$. Столь же очевидна инъективность отображения $f_{1}$, что приводит к ацикличности в члене $\mathfrak{e}(3)$ и по симметрии в $\mathfrak{e}(3)^{*}$.

2.2. Кручение и инвариант. Кручение комплекса (3) зададим формулой

$$
\tau \stackrel{\text { def }}{=} \frac{\text { minor } f_{1} \cdot \operatorname{minor} f_{3} \cdot \operatorname{minor} f_{5}}{\operatorname{minor} f_{2} \cdot \operatorname{minor} f_{4}},
$$

где миноры матриц выбираются согласно стандартному определению невырожденной $\tau$-цепи [12], если она существует. Поясним это подробнее, а также опишем наш выбор миноров в случае, когда невырожденной $\tau$-цепи не существует.

Выбор $\tau$-цепи - это выбор нескольких базисных векторов из фиксированного базиса каждого векторного пространства в алгебраическом комплексе ${ }^{3)}$. Эти базисные векторы соответствуют строкам, которые мы выбираем для очередного минора, остальные базисные векторы будут соответствовать столбцам следующего минора, и так далее. Невырожденной называется $\tau$-цепь, для которой все миноры отличны от нуля; для ациклического комплекса такая $\tau$-цепь всегда существует. Мы хотим, однако, использовать формулу (5) даже при отсутствии ацикличности. Из замечания 2 нетрудно вывести, что всегда существует $\tau$-цепь, для которой все миноры в формуле (5) не равны нулю, кроме, возможно, minor $f_{3}$. Именно так мы и будем всегда выбирать нашу $\tau$-цепь.

Кручение (5) обратно кручению, определенному в соответствии с работой [4] и другими нашими предшествующими работами, это же относится и к вводимому ниже инварианту $I$. Причина перехода к новому определению в том, что ниже в теоремах 5 и 6 мы будем иметь дело с линейными комбинациями именно таких кручений, как (5), точнее, их аналогов (9) для многообразий с краем. Отметим также, что если комплекс (3) (или комплекс (8), введенный ниже) неацикличен, то в соответствии с нашим определением (5) получится $\tau=0$, а по старому определению получилось бы бесконечное кручение.

Простая адаптация теоремы 1 из работы [4] (и небольшое уточнение, касающееся правильной расстановки знаков) дает следующую теорему.

Tеорема 2. Величина

$$
I(M)=\frac{\tau \prod(-6 V)}{\prod l^{2}},
$$

где произведение в числителе вычисляется по всем тетраэдрам, а в знаменателе по всем ребрам, является инвариантом многообразия $M$.

\footnotetext{
3) Мы с самого начала считаем все векторные пространства пространствами векторов-столбцов, и это означает, разумеется, что в них выбраны фиксированные базисы.
} 
ЗАмЕчАниЕ 3. Симметрия комплекса (3) снимает проблему знака кручения и, соответственно, инварианта $I(M)$ : кручение определяется однозначно, если взять minor $f_{3}$ диагональным (т.е. выбрать строки с теми же номерами, что и столбцы), a minor $f_{4}$ и minor $f_{5}$ выбрать симметричными minor $f_{2}$ и minor $f_{1}$ соответственно. Именно такой выбор подразумевается в теореме 2.

\section{3. АЛГЕБРАИЧЕСКИЙ КОМПЛЕКС И ВЕКТОР ИНВАРИАНТОВ ДЛЯ МНОГОБРАЗИЯ С ТРИАНГУЛИРОВАННЫМ КРАЕМ}

Пусть теперь $M$ - трехмерное компактное ориентируемое многообразие с краем, причем на крае задана фиксированная триангуляция, а внутри многообразия триангуляцию разрешено менять. При построении аналога комплекса (3) нужно учитывать, что вершины и ребра делятся на внутренние и лежащие на границе (ребро считается граничным, только если оно целиком лежит на границе). Для граничных ребер не определен угол дефекта (4), поэтому вместо него возьмем максимально похожую на него величину

$$
\alpha_{i} \stackrel{\text { def }}{=}-\sum_{k} \varphi_{i}^{(k)}(\bmod 2 \pi),
$$

т.е. двугранный угол при данном ребре, взятый со знаком минус. Кроме того, желая получить как можно больше инвариантов, мы выберем и пока фиксируем два произвольных подмножества $\mathcal{C}$ и $\mathcal{D}$ одинаковой мощности из множества граничных ребер.

Определим следующую укороченную модификацию комплекса (3):

$$
0 \longrightarrow\left(d x_{\text {внутр }}\right) \stackrel{f_{2}}{\longrightarrow}\left(d l_{\text {внутр }}, d l_{\text {гран }}\right) \stackrel{f_{3}}{\longrightarrow}(d \omega, d \alpha) \stackrel{f_{4}=-f_{2}^{\mathrm{T}}}{\longrightarrow}\left(d x_{\text {внутр }}^{*}\right) \longrightarrow 0 .
$$

В нашем комплексе теперь участвуют только дифференциалы координат внутрен-

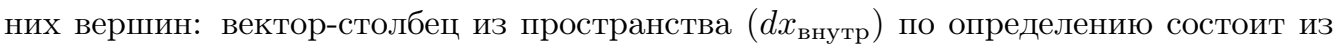
этих дифференциалов и только из них. Далее, по определению вектор-столбец из пространства $\left(d l_{\text {внутр }}, d l_{\text {гран }}\right)$ состоит из дифференциалов длин всех внутренних ребер, а также граничных ребер, входящих в множество $\mathcal{C}$. Вектор-столбец из пространства $(d \omega, d \alpha)$ по определению состоит из дифференциалов углов дефекта для всех внутренних ребер и дифференциалов $d \alpha_{i}$ для ребер $i \in \mathcal{D}$. Наконец, в пространстве $\left(d x_{\text {внутр }}^{*}\right)$ мы также задействуем величины, относящиеся ко всем внутренним вершинам, и только их ${ }^{4)}$.

ТЕорема 3. Последовательность (8) является алгебраическим комплексом при любом выборе множеств $\mathcal{C}$ и $\mathcal{D}$.

Доказательство. Равенство $f_{3} \circ f_{2}=0$ следует из таких же геометрических соображений, как и в доказательстве теоремы 1. Нужно только обратить внимание, что не только углы дефекта остаются равными нулю при движениях внутренних

\footnotetext{
4) Мы определили в разделе 2 пространство $\left(d x^{*}\right)$ как пространство векторов-столбцов, не вдаваясь в их геометрический смысл. Тем не менее каждый элемент столбца очевидным образом относится к определенной вершине.
} 
вершин, но и двугранные углы $\alpha$ при граничных ребрах не меняются, коль скоро граничные вершины неподвижны.

Равенство $f_{4} \circ f_{3}=0$ следует из предыдущего, если сделать взаимную замену $\mathcal{C} \leftrightarrow \mathcal{D}$ и транспонировать матрицы. Теорема доказана.

Если комплекс (8) ацикличен, то определим его кручение формулой

$$
\tau \stackrel{\text { def }}{=} \frac{\operatorname{minor} f_{3}}{\operatorname{minor} f_{2} \cdot \operatorname{minor} f_{4}},
$$

где миноры выбираются согласно правилу для невырожденной $\tau$-цепи и, кроме того, minor $f_{2}$ и minor $f_{4}$ должны быть симметричны друг другу. Даже если комплекс (8) неацикличен, у прямоугольной матрицы $f_{2}$ всегда существует ненулевой минор максимального размера (равного утроенному числу внутренних вершин): это легко понять, если перечислять внутренние вершины в определенном порядке, всякий раз сопоставляя вершине три ребра. Начинаем с вершины, соединенной (как минимум) тремя ребрами с границей, и включаем в minor $f_{2}$ три столбца, соответствующие этой вершине, и три строки, соответствующие этим ребрам; дальше на каждом шаге выбираем вершину, соединенную с границей и/или уже выбранными вершинами тремя ребрами, и поступаем аналогично. Полученный минор имеет блочно-треугольный вид (на диагонали стоят блоки размера $3 \times 3$ ), и не составляет труда убедиться, что он не равен нулю (напомним, что координаты всех вершин находятся в общем положении). Будем поэтому считать, что именно такой minor $f_{2}$ и симметричный ему minor $f_{4}$, одинаковые для всех $\mathcal{C} u \mathcal{D}$, выбираются в формуле $(9)$, a для minor $f_{3}$ берутся строки и столбцы, соответствующие ребрам, не задействованным в minor $f_{4}$ и minor $f_{2}$ соответственно. Это распространяет определение (9) на неациклический случай minor $f_{3}=0$.

Tеорема 4. Величина

$$
I_{\mathcal{C}, \mathcal{D}}(M)=\frac{\tau \prod(-6 V)}{\prod l^{2}},
$$

где в числителе произведение вычисляется по всем тетраэдрам, а в знаменателе по внутренним ребрам, для данных множеств граничных ребер $\mathcal{C}$ и $\mathcal{D}$ является инвариантом многообразия $M$ с триангулированным краем и представляет собой функцию координат граничных вериин.

Доказательство. Мощность каждого из множеств $\mathcal{C}$ и $\mathcal{D}$ будем называть уровнем соответствующего инварианта. Например, пустым множествам соответствует инвариант нулевого уровня, именно такие инварианты изучались в работе [10]. В этой работе изложено доказательство аналогичного утверждения для частного случая инвариантов нулевого уровня и тора со специальной триангуляцией в качестве края многообразия. Напомним, что доказательство состоит из двух основных частей: сначала мы доказываем, что от одной триангуляции к другой можно перейти "относительными", т.е. не затрагивающими границу, движениями Пахнера $2 \leftrightarrow 3$ и $1 \leftrightarrow 4$, а затем исследуем, что́ происходит с матрицами, входящими в комплекс, при этих движениях. Несмотря на то что сейчас мы находимся в более общей ситуации, рассуждения из работы [10] справедливы и в нашем случае, так что мы отсылаем читателя к указанной работе за подробностями. 
ЗАмечАние 4 . Вопрос о знаке инвариантов $I_{\mathcal{C}, \mathcal{D}}(M)$ чуть сложнее, чем в случае замкнутых многообразий: minor $f_{5}$ и minor $f_{4}$ по-прежнему симметричны minor $f_{1}$ и minor $f_{2}$ соответственно, но знак minor $f_{3}$ при $\mathcal{C} \neq \mathcal{D}$ зависит от упорядочения ребер. Нетрудно видеть, однако, что достаточно задать упорядочение только граничных ребер, так что эта зависимость не вносит особых трудностей.

Разумеется, всевозможные инварианты для разных $\mathcal{C}, \mathcal{D}$ далеко не независимы; подробно мы обсуждаем это в разделе 7.

\section{4. ПРЕОБРАЗОВАНИЕ ВЕКТОРА ИНВАРИАНТОВ ПРИ ИЗМЕНЕНИИ ТРИАНГУЛЯЦИИ КРАЯ}

Рассмотрим, что происходит с набором инвариантов (10), взятых для всевозможных $\mathcal{C}, \mathcal{D}$, при изменении триангуляции края $\partial M$ многообразия $M$. От одной триангуляции $\partial M$ к другой можно перейти с помощью последовательности двумерных движений Пахнера $2 \rightarrow 2$ и $1 \leftrightarrow 3$. Движение $2 \rightarrow 2$ соответствует приклеиваниюк краю нового тетраэдра по двум граням, например тетраэдра $A B C D$ по граням $A B C$ и $B D C$. Ребро $B C$ становится внутренним, вместо него на границе появляется новое ребро $A D$. Выберем какие-то множества ребер $\mathcal{C}$ и $\mathcal{D}$ после движения $2 \rightarrow 2$ и попробуем выразить миноры, входящие в формулу (9), через соответствующие миноры, выбранные до этого движения.

Прежде всего, мы сохраним те же миноры матриц $f_{2}$ и $f_{4}$, которые выбраны так, как описано после формулы (9), поскольку нужные для них строки и столбцы этих матриц не претерпели изменений (напомним, что эти миноры к тому же не зависят от $\mathcal{C}$ и $\mathcal{D})$. Теперь изучим, что́ происходит с матрицей $f_{3}$.

Рассмотрим сначала "полную" матрицу $f_{3}$, которая соответствует случаю $\mathcal{C}=\mathcal{D}=\{$ все граничные ребра $\}$. $\mathrm{C}$ ней происходят такие изменения: добавим к $f_{3}$ сначала нулевые строку и столбец, которые будут соответствовать ребру $A D$. Затем прибавим к полученной матрице матрицу $\left(\partial \alpha_{i} / \partial l_{j}\right)$ производных двугранных углов, взятых со знаком минус, по длинам ребер в новом тетраэдре $A B C D$, также дополненную необходимыми нулевыми строками и столбцами.

Что касается матрицы minor $f_{3}$ для произвольных $\mathcal{C}, \mathcal{D}$, то, будучи подматрицей "полной" матрицы, она получается из некоторой подматрицы "полной" матрицы, взятой до движения $2 \rightarrow 2$, аналогичным способом: добавлением, если необходимо, новых строки и столбца и прибавлением нужной подматрицы $\left(\partial \alpha_{i} / \partial l_{j}\right)$. Ключ к вычислению нового минора дает следующая простая лемма.

Лемма 1. Определитель суммы матрии, $A+B$ равен

$$
\operatorname{det}(A+B)=\sum \epsilon \cdot \operatorname{minor} A \cdot \operatorname{minor} B
$$

где суммирование происходит по всем минорам матрицы $A$, от нулевого минора до минора полного размера; для minor $B$ берутся строки и столбцы, дополнительные $\kappa$ minor $A$; знак $\epsilon= \pm 1$ соответствует знаку при алгебраическом дополнении $\kappa \operatorname{minor} A$. 
ДокАзАтЕЛЬство. Запишем $\operatorname{det}(A+B)$ как сумму с чередующимися знаками произведений элементов матрицы $A+B$ и раскроем скобки в каждом члене. Очевидно, полученная сумма состоит в точности из тех же слагаемых, что и правая часть (11). Лемма доказана.

Считая, что матрица нового minor $f_{3}$ играет роль матрицы $A+B$ в лемме 1 , а нужная подматрица $\left(\partial \alpha_{i} / \partial l_{j}\right)$ для тетраэдра $A B C D$ - роль $A$, получаем линейную комбинацию величин minor $B$, причем нетрудно убедиться, что каждый из этих миноров, входящих в сумму (11) с ненулевыми коэффициентами, является в точности minor $f_{3}$ из формулы $(9)$ для некоторых $\mathcal{C}, \mathcal{D}$ в ситуации до движения $2 \rightarrow 2$.

Рассматривая инварианты $(10)$ для различных $\mathcal{C}, \mathcal{D}$ как компоненты вектора инвариантов $\vec{I}(M)$, получаем следующую лемму.

Лемма 2. Движению Пахнера $2 \rightarrow 2$ на крае многообразия $M$ соответствует линейное преобразование вектора инвариантов $\vec{I}(M)$.

ДокАЗАТЕЛьство. Нам остается заметить, что новый множитель вида $(-6 V) / l^{2}$ в формуле (10), вызванный появлением нового тетраэдра и нового внутреннего ребра, не нарушает линейности преобразования набора миноров $f_{3}$ и, следовательно, вектора $\vec{I}(M)$. Лемма доказана.

Аналогичный результат получается и для движений $1 \leftrightarrow 3$. Движение $1 \rightarrow 3$ отличается от $2 \rightarrow 2$ с этой точки зрения только способом приклеивания нового тетраэдра: он приклеивается по одной грани. Рассмотрим чуть более сложный случай $3 \rightarrow 1$. Пусть в триангуляции края имелся треугольник $A B C$, разбитый на три треугольника $A B D, B C D$ и $C A D$. Движению $3 \rightarrow 1$ соответствует приклеивание тетраэдра $D A B C$ по этим трем граням. При этом вершина $D$ и ребра $A D, B D$ и $C D$ становятся внутренними. Поэтому к матрице $f_{2}$ добавляются три столбца, соответствующие частным производным по $x_{D}, y_{D}$ и $z_{D}$. Соответственно, в minor $f_{2}$ в формуле (9) также с необходимостью будут содержаться три этих столбца, а также три строки, соответствующие длинам $l_{A D}, l_{B D}$ и $l_{C D}$. Стандартные соображения, связанные с блочной треугольностью, показывают, что в результате minor $f_{2}$ просто умножается на определитель матрицы Якоби от трех указанных длин по трем координатам $D$; это же относится и к minor $f_{4}$. Такое умножение не нарушает линейности, так что мы имеем еще одну лемму.

Лемма 3. Движениям Пахнера $1 \leftrightarrow 3$ на крае многообразия $M$ соответствуют линейные преобразования вектора инвариантов $\vec{I}(M)$.

Из лемм 2 и 3 вытекает следующая теорема.

Теорема 5. Переходу от одной триангулячии края компактного трехмерного многообразия $M \kappa$ другой его триангулячии можно сопоставить линейное преобразование $\mathcal{A}$ такое, что вектор инвариантов $\vec{I}(M)$ переходит в $\mathcal{A} \vec{I}(M)$, причем $\mathcal{A}$ можно выбрать зависящим только от начальной и конечной триангуляций края, но не от самого $M$. 
ДокАзАтельство. Рассмотрим цепочку движений Пахнера, переводящую старую триангуляцию края в новую. Согласно предыдущим рассуждениям приклеивание соответствующей последовательности тетраэдров определяет цепочку линейных преобразований, действующих на $\vec{I}(M)$.

ЗАмЕчАниЕ 5. Мы не доказали, что две различные цепочки движений Пахнера, приводящие к одному и тому же преобразованию триангуляции края, дадут одно и то же $\mathcal{A}$. Даже если бы это оказалось не так, новые векторы $\mathcal{A} \vec{I}(M)$ при любом фиксированном $M$ и предполагаемых различных $\mathcal{A}$ обязаны совпадать. Наша гипотеза состоит в том, что изложенный выше алгоритм построения $\mathcal{A}$ приводит к результату, не зависящему от выбора конкретной цепочки.

\section{5. СКЛЕИВАНИЕ МНОГООБРАЗИЙ И КОМПОЗИЦИЯ ИНВАРИАНТОВ}

Пусть $M_{1}$ и $M_{2}$ - компактные ориентированные трехмерные многообразия с одинаковыми, но противоположно ориентированными однокомпонентными краями: $\partial M_{1}=-\partial M_{2}$. Пусть также на $\partial M_{1}$ и $\partial M_{2}$ заданы одинаковые триангуляции, так что $M_{1}$ и $M_{2}$ можно склеить, отождествив соответствующие друг другу симплексы их краев. Результатом этого склеивания будет замкнутое многообразие $M$.

Выберем для $M_{1}$ и $M_{2}$ триангуляции, ограничение которых на край приводит к заданной на нем триангуляции. Таким образом, $M$ также оказывается триангулированным; рассмотрим для него алгебраический комплекс (3). Вычислим кручение этого комплекса, выбрав $\tau$-цепь (миноры) так, чтобы данное кручение можно было связать с кручениями комплексов (8) для $M_{1}$ и $M_{2}$.

Нам понадобится следующее техническое условие на триангуляцию поверхности $\Gamma \subset M$ - общего края $M_{1}$ и $M_{2}$, рассматриваемого безотносительно к его ориентации.

УсловиЕ. Вершины поверхности $\Gamma$ можно записать в таком порядке $A, B, C, D, \ldots, Z$, что, во-первых, вершины $A, B$ и $C$ образуют треугольник, т.е. соединены друг с другом ребрами; во-вторых, начиная с $D$ каждая вершина соединена ребрами по меньшей мере с тремя предыдущими вершинами (все ребра предполагаются лежащими в Г).

Триангуляция Г, удовлетворяющая данному условию, несомненно, существует, ее легко построить для поверхности Г любого рода $g$. Представим $Г$ стандартным образом в виде $4 g$-угольника со следующими сторонами в порядке обхода в положительном направлении: $a_{1}, b_{1}, a_{1}^{-1}, b_{1}^{-1}, \ldots, a_{g}, b_{g}, a_{g}^{-1}, b_{g}^{-1}$. Все вершины этого многоугольника, как известно, отождествляются, обозначим получающуюся вершину через $D$. Еще одну вершину $C$ расположим в центре многоугольника, и $2 g$ вершин будут находиться на серединах сторон многоугольника в следующем порядке: $A, B, A, B, E, F, E, F, \ldots$. Наконец, соединим центры сторон последовательно друг с другом, а также с центром многоугольника (см. рис. 1). Далее мы считаем, что для Г выбрана триангуляция, удовлетворяющая указанному выше условию, например та, что изображена на рис. 1. 


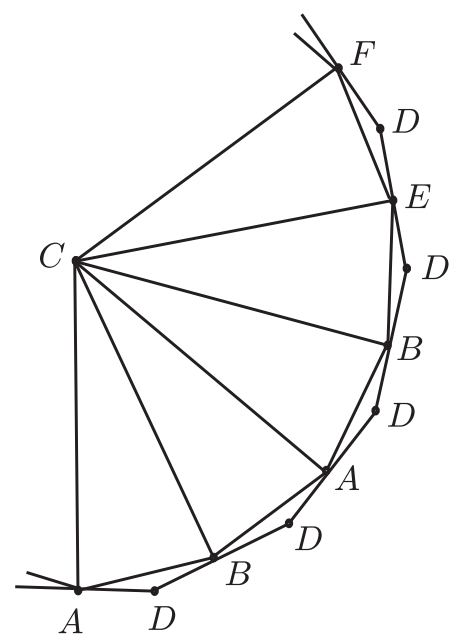

Рис. 1. Стандартная триангуляция общего края Г многообразий $M_{1}$ и $M_{2}$.

Упорядочим и обозначим вершины $Г$, как сказано в условии. Возьмем для минора матрицы $f_{1}$ ее строки, соответствующие дифференциалам координат $d x_{A}, d y_{A}$, $d z_{A}, d y_{B}, d z_{B}, d z_{C}$. Симметрично поступим с $f_{5}$, т.е. возьмем для минора этой матрицы столбцы, соответствующие тем же дифференциалам.

Теперь займемся minor $f_{2}$, строки которого соответствуют ребрам триангуляции или, более точно, дифференциалам их длин. Что касается ребер из Г, включим в minor $f_{2}$ строки, соответствующие $d l_{A B}, d l_{B C}, d l_{C A}$, а также по три строки для каждой из следующих вершин $D, \ldots, Z$; эти строки соответствуют ребрам, упомянутым в условии. Кроме того, включим в minor $f_{2}$ для $M$ все строки, находившиеся в аналогичных минорах, по которым мы вычисляли кручения (9) для $M_{1}$ и $M_{2}$. Напомним, что способ построения этих миноров считается фиксированным и объяснен после формулы (9). Далее, разумеется, выберем minor $f_{4}$ симметричным minor $f_{2}$.

Сохраним обозначение minor $f_{2}$ для минора, относящегося к комплексу (3) для $M$, а для миноров, относящихся к комплексам (8) для $M_{1}$ и $M_{2}$, введем обозначения $\left(\operatorname{minor} f_{2}\right)^{(1)}$ и $\left(\operatorname{minor} f_{2}\right)^{(2)}$ соответственно. Тогда

$$
\text { minor } f_{2}=\left(\operatorname{minor} f_{2}\right)^{(1)} \cdot\left(\operatorname{minor} f_{2}\right)^{(2)} \cdot\left(\operatorname{minor} f_{2}\right)^{\Gamma} \text {, }
$$

где

$$
\left(\operatorname{minor} f_{2}\right)^{\Gamma}=\frac{d l_{A B} \wedge d l_{B C} \wedge d l_{C A}}{d x_{B} \wedge d x_{C} \wedge d y_{C}} \cdot \frac{d l_{A D} \wedge d l_{B D} \wedge d l_{C D}}{d x_{D} \wedge d y_{D} \wedge d z_{D}} \cdot \ldots
$$

- множитель в minor $f_{2}$, возникающий на границе. Мы позволили себе записать определители матриц Якоби (размера $3 \times 3$ ) в виде отношений внешних произведений дифференциалов. Разумеется, равенства (12), (13) получаются с использованием все той же блочной треугольности матриц, а многоточие в конце (13) подразумевает, что следующий множитель содержит в знаменателе дифференциалы трех координат следующей вершины $E$, а в числителе - дифференциалы длин трех ребер, которыми $E$ соединена с предыдущими вершинами согласно условию, и так далее. 
Аналогично мы сохраним обозначение minor $f_{3}$ для минора, относящегося к комплексу (3) для $M$, а для миноров, относящихся к комплексам (8) для $M_{1}$ и $M_{2}$, введем обозначения $\left(\operatorname{minor} f_{3}\right)_{\mathcal{C}_{1}, \mathcal{D}_{1}}^{(1)}$ и $\left(\operatorname{minor} f_{3}\right)_{\mathcal{C}_{2}, \mathcal{D}_{2}}^{(2)}$ соответственно, где $\mathcal{C}_{i}, \mathcal{D}_{i}$ - множества ребер $\mathcal{C}$ и $\mathcal{D}$ (введенные в начале раздела 3 ) для каждого $M_{i}, i=1,2$. Чтобы вычислить minor $f_{3}$, мы снова используем лемму 1. Получим

$$
\text { minor } f_{3}=\sum_{\mathcal{C}_{1}, \mathcal{D}_{1}} \epsilon \cdot\left(\operatorname{minor} f_{3}\right)_{\mathcal{C}_{1}, \mathcal{D}_{1}}^{(1)} \cdot\left(\operatorname{minor} f_{3}\right)_{\overline{\mathcal{C}}_{1}, \overline{\mathcal{D}}_{1}}^{(2)},
$$

где $\overline{\mathcal{C}}_{1}$ и $\overline{\mathcal{D}}_{1}$ - дополнения множеств $\mathcal{C}_{1}$ и $\mathcal{D}_{1}$ соответственно в множестве $\mathcal{E}$ всех ребер $\Gamma$, из которого исключены ребра, упомянутые в условии и уже задействованные для minor $f_{2}$ и симметричного ему minor $f_{4}$. Таким образом, $\mathcal{E}$ состоит из ребер $\Gamma$, кроме $A B, B C, C A, A D, B D, C D$ и так далее по три ребра на каждую новую вершину.

Теорема 6. Инвариант $I(M)$ замкнутого многообразия $M$, полученного склеиванием двух многообразий $M_{1}$ и $M_{2}$ с однокомпонентным краем, равен следующему скалярному произведению векторов инвариантов $\vec{I}\left(M_{1}\right)$ и $\vec{I}\left(M_{2}\right)$ :

$$
I(M)=c \sum_{\mathcal{C}_{1}, \mathcal{D}_{1}} \epsilon I_{\mathcal{C}_{1}, \mathcal{D}_{1}}\left(M_{1}\right) I_{\overline{\mathcal{C}}_{1}, \overline{\mathcal{D}}_{1}}\left(M_{2}\right),
$$

где величина с относится только к краю Г, по которому идет склеивание:

$$
\begin{aligned}
c & =\frac{\left(\operatorname{minor} f_{1}\right)^{2}}{(-1)^{s}\left(\left(\operatorname{minor} f_{2}\right)^{\Gamma}\right)^{2} \prod l^{2}}= \\
& =\frac{(-1)^{s}}{\prod l^{2}}\left(l_{A B} l_{B C} l_{C A} \cdot \frac{l_{A D} l_{B D} l_{C D}}{6 V_{A B C D}} \cdot \ldots\right)^{2}=\frac{(-1)^{s}}{\prod_{i \in \mathcal{E}} l_{i}^{2} \cdot\left(6 V_{A B C D} \cdot \ldots\right)^{2}},
\end{aligned}
$$

где произведение $\prod l^{2}$ вычисляется по ребрам из $\Gamma, s$ - число строк в $\left(\operatorname{minor} f_{2}\right)^{\Gamma}$. Каждый из сомножителей, обозначенных многоточием в левой части второй строки формуль (16), аналогичен $l_{A D} l_{B D} l_{C D} / 6 V_{A B C D}$, но вместо $D$ для очередного сомножителя берется очередная вершина согласно приведенному выше условию, а вместо $A, B$ и $C$ - три вершины, с которыми она соединена согласно тому же условию.

ДокАЗАтЕЛьство. Формула (15) вместе с первым равенством в формуле (16) получается из формул (5), (6), (9), (10), (12) и (14) с учетом равенства minor $f_{1}=$ minor $f_{5}$, а также равенства с точностью до знака $(-1)^{s}$ minor $f_{2}^{\Gamma}$ и minor $f_{4}^{\Gamma}$. Кстати, из того, что $s$ - это число ребер, упоминаемых в условии, нетрудно вывести, что

$$
s=3 \cdot(\text { число вершин в } Г)-6 .
$$

Второе равенство в (16) получается прямым вычислением миноров: для minor $f_{1}-$ с использованием формул, показывающих, как элементы $(3)$, т.е. три бесконечно малых сдвига и три поворота, действуют на координаты $x_{A}, y_{A}, z_{A}, y_{B}, z_{B}, z_{C}$, а для $\left(\operatorname{minor} f_{2}\right)^{\Gamma}$ - с использованием формулы (13). Третье равенство в (16) получается по определению множества $\mathcal{E}$ после сокращения квадратов длин. Разумеется, 
объем $V_{A B C D}$ и следующие, скрывающиеся за многоточиями, имеют чисто формальный смысл и могут не соответствовать никаким тетраэдрам из триангуляции, ибо точки $A, B, C, D, \ldots$ лежат на поверхности $\Gamma$.

ЗАмечАниЕ 6. Знак $\epsilon$ в формулах (14) и (15) тоже, разумеется, зависит только от информации, относящейся к Г. Именно, $\epsilon=(-1)^{\sigma}$, где $\sigma$ - сумма номеров всех элементов $\mathcal{C}_{1}$ и $\mathcal{D}_{1}$ при данном упорядочении вершин $\Gamma$, ср. с замечанием 4.

\section{6. ПРИМЕРЫ}

6.1. Полноторие. Пусть $M$ - полноторие и, соответственно, его край $\partial M-$ двумерный тор. Склеиваем полноторие из шести тетраэдров следующим образом. Сначала берем два одинаковых тетраэдра $A B C D$ и склеиваем их по ребрам: ребро $A D$ одного тетраэдра приклеиваем к ребру $A D$ другого и аналогично поступаем с ребрами $B C$. Возникает цепочка из двух тетраэдров; быть полноторием ей мешает "нулевая толщина" в местах склеек. Представим себе, что эта цепочка расположена так, как показано на рис. 2. Приклеим с "нашей" стороны еще один тетраэдр $D A B C$ (противоположной ориентации!) к граням $A D C$ и $A B D$. Это создаст ненулевую толщину около ребра $A D$. Приклеим еще один тетраэдр $A B C D$ к двум свободным граням нового тетраэдра $D A B C$, т.е. к $A B C$ и $B D C$, для того чтобы получить триангуляцию $\partial M$, изображенную на рис. 4. Совершенно аналогично, чтобы убрать нулевую толщину вдоль ребра $B C$, приклеим еще один тетраэдр противоположной ориентации с "нашей" стороны рисунка к граням $B A C$ и $C D B$, а затем к двум свободным граням нового тетраэдра приклеим тетраэдр “обычной” ориентации.

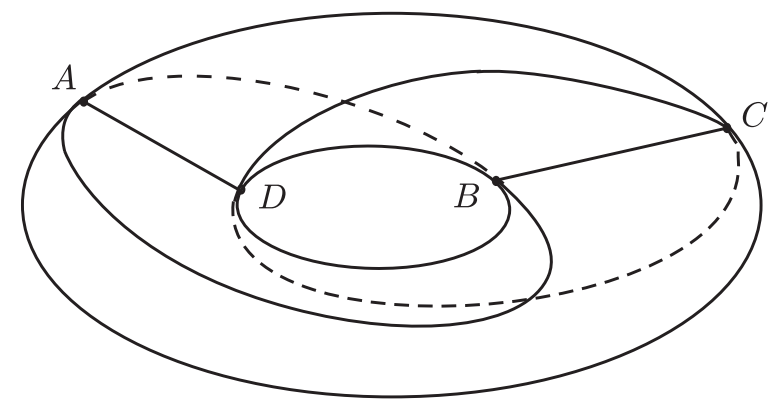

Рис. 2. Начало построения триангулированного полнотория - цепочка из двух тетраэдров $A B C D$.

Чтобы различать одноименные ребра, введем следующие обозначения. Ребра $A D$ и $B C$, присутствующие на рис. 2, обозначим $A D_{0}$ и $B C_{0}$. Далее считаем один из тетраэдров на рис. 2 первым, а другой - вторым и в соответствии с этим присвоим их остальным ребрам индексы 1 или 2. Остается обозначить еще четыре ребра, два из которых лежат внутри полнотория (кроме, разумеется, их концов), а два - на границе. Внутренние ребра обозначим $A D_{3}$ и $B C_{3}$, а граничные $-A D_{4}$ и $B C_{4}$. 


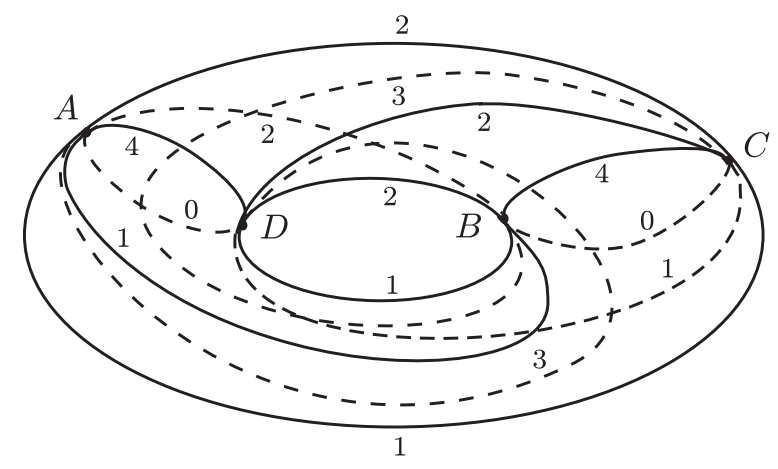

Рис. 3. Триангулированное полноторие.

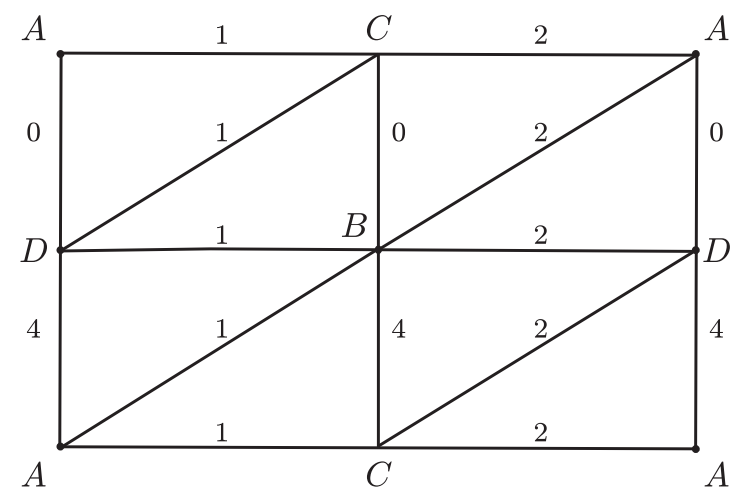

Рис. 4. Развертка триангуляции тора.

Получившееся триангулированное полноторие изображено на рис. 3, а развертка получившейся триангуляции тора (его границы) - на рис. 4. На этих рисунках цифры соответствуют введенным выше индексам ребер. "Полная" матрица $f_{3}$ имеет размер $14 \times 14$. Ее матричные элементы можно тем не менее представить в обозримом виде, если выписывать не их самих, а их отношения к стандартным выражениям, представляющим собой частные производные взятых со знаком минус двугранных углов $\alpha_{i}$ по длинам $l_{j}$ в тетраэдре $A B C D$, где $i$ и $j$-ребра с теми же именами, что у матрицы $f_{3}$, только без индексов. Эти отношения представлены в таблице ${ }^{5)}$.

Инвариант нулевого уровня соответствует $(2 \times 2)$-минору полной матрицы $f_{3}$, получающемуся из двух последних строк и двух последних столбцов таблицы. Так как этот минор полностью состоит из нулей, не только этот инвариант, но и все инварианты первого уровня равны нулю. Большинство инвариантов второго уровня тоже равны нулю, а ненулевые характеризуются тем, что оба множества $\mathcal{C}$ и $\mathcal{D}$ состоят из половинок меридианов полнотория. Таким образом, наши инварианты

\footnotetext{
5) Метод вычисления элементов таблицы прост: смотрим, какие из шести тетраэдров триангуляции являются общими для двух данных ребер. Каждому тетраэдру сопоставляем число \pm 1 в соответствии с его ориентацией и суммируем эти числа.
} 
ТАБлицА. Коэффициенты, на которые умножаются производные $\partial \alpha_{i} / \partial l_{j}$, взятые в одном тетраэдре $A B C D$; эти коэффициенты необходимы для получения элементов матрицы $f_{3}$ для полнотория.

\begin{tabular}{c|cccccccccccccc} 
& $A C_{1}$ & $A B_{1}$ & $A D_{4}$ & $B D_{1}$ & $C D_{1}$ & $A D_{0}$ & $A C_{2}$ & $C D_{2}$ & $B C_{4}$ & $B D_{2}$ & $A B_{2}$ & $B C_{0}$ & $B C_{3}$ & $A D_{3}$ \\
\hline$A C_{1}$ & 1 & 1 & 0 & 1 & 1 & 1 & 0 & 0 & 1 & 0 & 0 & 0 & 0 & 0 \\
$A B_{1}$ & 1 & 1 & 1 & 1 & 1 & 0 & 0 & 0 & 1 & 0 & 0 & 0 & 0 & 0 \\
$A D_{4}$ & 0 & 1 & 1 & 1 & 0 & 0 & 1 & 1 & 0 & 0 & 0 & 0 & 1 & 0 \\
$B D_{1}$ & 1 & 1 & 1 & 1 & 1 & 0 & 0 & 0 & 0 & 0 & 0 & 1 & 0 & 0 \\
$C D_{1}$ & 1 & 1 & 0 & 1 & 1 & 1 & 0 & 0 & 0 & 0 & 0 & 1 & 0 & 0 \\
$A D_{0}$ & 1 & 0 & 0 & 0 & 1 & 1 & 0 & 0 & 0 & 1 & 1 & 2 & -1 & 0 \\
$A C_{2}$ & 0 & 0 & 1 & 0 & 0 & 0 & 1 & 1 & 0 & 1 & 1 & 1 & 0 & 0 \\
$C D_{2}$ & 0 & 0 & 1 & 0 & 0 & 0 & 1 & 1 & 1 & 1 & 1 & 0 & 0 & 0 \\
$B C_{4}$ & 1 & 1 & 0 & 0 & 0 & 0 & 0 & 1 & 1 & 1 & 0 & 0 & 0 & 1 \\
$B D_{2}$ & 0 & 0 & 0 & 0 & 0 & 1 & 1 & 1 & 1 & 1 & 1 & 0 & 0 & 0 \\
$A B_{2}$ & 0 & 0 & 0 & 0 & 0 & 1 & 1 & 1 & 0 & 1 & 1 & 1 & 0 & 0 \\
$B C_{0}$ & 0 & 0 & 0 & 1 & 1 & 2 & 1 & 0 & 0 & 0 & 1 & 1 & 0 & -1 \\
$B C_{3}$ & 0 & 0 & 1 & 0 & 0 & -1 & 0 & 0 & 0 & 0 & 0 & 0 & 0 & 0 \\
$A D_{3}$ & 0 & 0 & 0 & 0 & 0 & 0 & 0 & 0 & 1 & 0 & 0 & -1 & 0 & 0
\end{tabular}

позволяют опознать меридианы полнотория, хотя на рис. 4 они присутствуют на равных основаниях с другими линиями.

ЗАмЕчАниЕ 7. Разумеется, склеивая два таких триангулированных полнотория по-разному, можно получить многообразия $S^{2} \times S^{1}$ или $S^{3}$, а если к тому же разрешить переходить к другим триангуляциям, то можно получить и все линзовые пространства. Мы оставляем исследование возникающих при этом алгебраических структур до следующих работ.

6.2. Линзовые пространства без трубчатой окрестности неузла. Напомним, что "неузлы" в линзовых пространствах $L(p, q)$, изучавшиеся в работе [10] это наименее заузленные петли, идущие вдоль нетривиальных элементов группы $\pi_{1}(L(p, q))$. В указанной работе вычислены инварианты многообразий с краем, получающихся удалением трубчатой окрестности такого неузла из $L(p, q)$. В терминах настоящей статьи это не что иное, как инварианты нулевого уровня, соответствующие различным триангуляциям края, которые сопоставлены в работе [10] различным оснащениям неузла.

Мы приведем здесь результаты, которые получаются по формулам статьи [10] для целочисленных оснащений неузлов в $L(7,1)$. Целочисленность оснащения в наших терминах означает, что край многообразия триангулирован, как на рис. 4 , причем меридиан $A D A$ (или $C B C$ ) фиксирован (в [10] рассматривались также оснащения, отличающиеся от данных на полоборота). Оснащения нумеруются числом $m \in \mathbb{Z}$; число $n=1,2,3$ указывает, сколько раз неузел обходит вокруг образующей фундаментальной группы ${ }^{6)}$. Ниже в формулах (17)-(20) приведены инварианты, получа-

\footnotetext{
6) За остальными подробностями - какому оснащению соответствует $m=0$, в какую сторону $m$ растет и в какую убывает, что конкретно выбирается в качестве образующей фундаментальной группы - мы отсылаем читателя к работе [10].
} 
ющиеся по формуле (10), с чуть измененными обозначениями: опущены подразумеваемые индексы $(\mathcal{C}, \mathcal{D})=(\varnothing, \varnothing)$, на их месте стоит число $n$; число $m$ стоит в верхнем индексе; эти числа вместе с названием линзового пространства определяют многообразие с краем и триангуляцию края.

Сначала отметим, что зависимость инварианта от координат, приписанных граничным вершинам, сводится к простому множителю:

$$
I_{n}^{(m)}(L(p, q))=\left(6 V_{A B C D}\right)^{4} \tilde{I}_{n}^{(m)}(L(p, q)),
$$

где величина с тильдой уже от этих координат не зависит (это верно для всех $p$ и $q$ ). Далее, из формул статьи [10] получаем следующее ${ }^{7)}$ :

$$
\begin{aligned}
& \tilde{I}_{1}^{(m)}(L(7,1))=-7^{2}(1-7 m)^{2}(6+7 m)^{2}, \\
& \tilde{I}_{2}^{(m)}(L(7,1))=-7^{2}(3-7 m)^{2}(10+7 m)^{2}, \\
& \tilde{I}_{3}^{(m)}(L(7,1))=-7^{2}(-5-7 m)^{2}(12+7 m)^{2} .
\end{aligned}
$$

Все числа, получаемые по данным формулам при всевозможных $m$, различны. Таким образом, для $L(7,1)$ наш инвариант нулевого уровня позволяет различить все неузлы со всеми оснащениями. Более того, он никогда не равен нулю, т.е. ведет себя совершенно иначе, чем в случае полнотория.

\section{7. ОБСУЖДЕНИЕ}

В настоящей статье сделан первый шаг к построению топологической теории поля в духе аксиом Атьи на основе кручений ациклических комплексов геометрической природы. Стремление построить такую теорию связано со следующими причинами.

Конечномерностъ: теория строится по конечной триангуляции многообразия, в ней нет континуальных интегралов.

Произвольная размерность многообразия: напомним еще раз о работах [4], [5], [11], в которых аналогичные геометрические кручения построены для четырехмерных многообразий.

Богатство и разнообразие возникающих теорий: в настоящей статье применялись алгебраические комплексы, использующие евклидову трехмерную геометрию; между тем уже проделанные нами расчеты [13] для геометрии, связанной с группой $\mathrm{SL}(2, \mathbb{C})$, дают существенно новые результаты.

Возможность введения q-коммутационных соотношений: одна из "неабелевых" разновидностей нашей теории, предложенная в работе [14], допускает редукцию, при которой некоммутирующие переменные удовлетворяют вейлевским соотношениям $b a=q a b$.

Нашей ближайшей целью видится, однако, обобщение конструкций, изложенных в настоящей статье, на многообразия с любым числом компонент края.

\footnotetext{
7) Величина $\tilde{I}_{n}^{(m)}(L(p, q))$ в настоящей статье соответствует величине $1 / I_{n}^{(2 m)}(L(p, q))$ в обозначениях работы [10].
} 
Завершим это обсуждение следующими замечаниями. Как указывалось в конце раздела 3, компоненты вектора инвариантов не независимы. Соотношения между ними вытекают из соотношений Сильвестра [15], [16] между минорами матрицы. Простейшее из соотношений Сильвестра имеет трехчленный билинейный вид и связывает определитель матрицы, минор, полученный вычеркиванием двух строк и двух столбцов, и четыре промежуточных минора. Оно напоминает известное "условие свободных фермионов" $a_{+} a_{-}+b_{+} b_{-}=c_{+} c_{-}$для шестивершинной модели статистической физики. Есть также другие указания на присутствие "свободных фермионов" в нашей теории. Чтобы объяснить, как получается, что некоторые из геометрических комплексов допускают введение $q$-коммутационных соотношений между входящими в них величинами, напомним, что в математической физике такое уже встречалось. В работе [17] рассматривались модели дискретной квантовой теории поля, которые, с одной стороны, отвечают свободным фермионам, а с другой, допускают $q$-коммутационные соотношения. Дело в том, что когда свободных фермионов “достаточно много", на них можно вводить дополнительные структуры.

Благодарности. Автор благодарит Р. М. Кашаева и Е. В. Мартюшева за плодотворные обсуждения. Работа выполнена при поддержке РФФИ (грант № 07-0196005-р_урал_а).

\section{Список литературы}

[1] M. F. Atiyah, Publ. Math. IHES, 68 (1988), 175-186.

[2] М. Атья, Геометрия и физика узлов, Мир, М., 1995.

[3] I. G. Korepanov, J. Nonlinear Math. Phys., 8:2 (2001), 196-210.

[4] И. Г. Корепанов, ТМФ, 133:1 (2002), 24-35.

[5] И. Г. Корепанов, ТМФ, 135:2 (2003), 179-195.

[6] I. G. Korepanov, E. V. Martyushev, J. Nonlinear Math. Phys., 9:1 (2002), 86-98.

[7] Е. В. Мартюшев, Изв. Челябинского науч. центра УрО РАН, 2003, № 2(19), 1-5.

[8] Е. В. Мартюшев, Изв. Челябинского науч. иентра УрО РАН, 2004, № 4(26), 1-5.

[9] Е. В. Мартюшев, Геометрические инварианты трехмерных многообразий, узлов и зацеплений, Дисс. ... канд. физ.-матем. наук, Южно-Уральский гос. ун-т, Челябинск, 2007.

[10] J. Dubois, I. G. Korepanov, E. V. Martyushev, Euclidean geometric invariant of framed knots in manifolds, arXiv: math/0605164.

[11] И. Г. Корепанов, ТМФ, 131:3 (2002), 377-388.

[12] В. Г. Тураев, Введение в комбинаторные кручения, МЦНМО, М., 2004.

[13] R. M. Kashaev, I. G. Korepanov, E.V. Martyushev, A finite-dimensional TQFT for three-manifolds based on group $\operatorname{PSL}(2, \mathbb{C})$ and cross-ratios, arXiv: 0809.4239.

[14] Р. М. Кашаев, Частное сообщение, 2007.

[15] В. В. Прасолов, Задачи и теоремь линейной алгебры, Наука, М., 1996.

[16] J. J. Sylvester, Philos. Magaz., Fourth Ser., 1 (1851), 295-305.

[17] Р. М. Кашаев, И. Г. Корепанов, С. М. Сергеев, ТМФ, 117:3 (1998), 370-384. 